\title{
EXPERIENCIAS EDUCATIVAS DE JÓVENES UNIVERSITARIOS CON NETFLIX: UN ESTUDIO MIXTO
}

\author{
EDUCATIONAL EXPERIENCES \\ OF YOUNG COLLEGE STUDENTS \\ WITH NETFLIX: A MIXED STUDY
}

Ligia García-Béjar ${ }^{1}$

https://orcid.org/0000-0002-2980-0314

Recibido: mayo 12, 2021 - Aceptado: mayo 31, 2021

\section{RESUMEN}

Este artículo presenta los resultados de la aplicación de la escala de engagement con medios digitales de David Mersey, Calder y Malthouse, la cual mide los distintos tipos de experiencias que una persona tiene al consumir contenidos en una plataforma digital. Este instrumento se empleó para medir experiencias de consumo con la plataforma Netflix, de 630 jóvenes universitarios mexicanos, entre las cuales resultaron relevantes las que satisfacen experiencias educativas entre este grupo de estudio. En la segunda fase se realizaron 8 grupos focales (46 estudiantes en total), en los cuales se corroboró que esta plataforma satisface necesidades más allá del entretenimiento. En resumen, los estudiantes utilizan Netflix también para aprender, particularmente para conocer cómo se representan distintos tipos de culturas, para obtener más conocimiento y contexto sobre temas de su interés, y para reflexionar sobre situaciones humanas representadas en los contenidos de la plataforma, e incluso usan contenidos de esta OTT como recurso y complemento educativo.

Palabras clave: Engagement educativo, Netflix, estudiantes universitarios, consumo de medios, México.

1 Doctorado en Comunicación, Universidad de los Andes, Chile. Especialidad en Competencias Didácticas, Universidad Panamericana. Secretaria de investigación, Escuela de Pedagogía, Universidad Panamericana, campus Guadalajara.ligarcia@up.edu.mx 


\begin{abstract}
This article presents the results of the application of the David Mersey, Calder and Malthouse digital media engagement scale, which measures the different types of experiences that a person has when consuming digital platforms. This instrument was used to measure consumer experiences with Netflix of 630 young Mexican university students, among which were relevant experiences that satisfy educational experiences among this study group. In the second phase of this study, 8 focus groups were conducted (46 students in total), in which it was corroborated that this platform satisfies needs beyond entertainment. In summary, students also use Netflix to learn, particularly to learn how different types of cultures are represented, to obtain more knowledge and context on topics of their interest and to reflect on human situations that are represented in the contents of the platform, and even use content from this OTT as a resource and educational complement.
\end{abstract}

Keywords: Learner Engagement, Netflix, Undergraduate Students, Media Consumption, México.

\title{
INTRODUCCIÓN
}

Una persona cualquiera ha pasado muchas horas de su vida consumiendo contenidos informativos y de entretenimiento; esta actividad se ha vuelto más relevante e incluso omnipresente en la vida de las personas, gracias a los cambios tecnológicos que permiten la convergencia digital, y la fragmentación de medios y de contenidos. Los aspectos tecnológicos han influido en el modo en el que se consume; los avances tecnológicos carecerían de impacto si las audiencias no respondieran positivamente a emplearlas, incluso cambiando usos, hábitos y costumbres de consumo. Es innegable la consideración de los aspectos sociales para entender el desarrollo de la industria de medios; particularmente porque las audiencias no son una masa uniforme, sino un conjunto de personas que evolucionan a lo largo de su ciclo vital de vida y manifiestan necesidades e intereses distintos de consumo (Albarran, 2018).

La digitalización de los medios de comunicación trajo fenómenos de consumo como la interactividad, el uso de multipantallas, el control de las audiencias de lo que se consume, cómo y dónde se consume. El empleo de medios de comunicación también responde a la cultura y al perfil sociodemográfico del entorno (Fernández-Beaumont, 2000). En este sentido, los hábitos y el estilo de vida de las audiencias jóvenes -y particularmente los universitarios-, se orienta a la apertura por la adquisición de nuevos modelos de consumo (Cruz-Sotelo et al., 2013) y experimentan placer por vivir una cotidianidad digitalizada. «Las redes sociales y los medios digitales se han convertido en recursos fundamentales de expresión e interacción social para ellos, pues a través de estos, realizan actividades lúdicas, educativas, informativas y se comunican con su entorno» (Domínguez y López, 2015, p. 50).

En el ecosistema digital de medios, muchas compañías han conseguido adaptarse a la digitalización de los contenidos y a la consolidación de estrategias multiplataforma, ya sea por la evolución de sus formas de distribución o por alianzas estratégicas; algunas otras han desaparecido o decrecido en el proceso y han surgido otras nuevas que han logrado colocarse de manera exitosa, e incluso de manera puntera, en el ecosistema de medios. En esta evolución, compañías digitales como Netflix, han surgido para establecerse como competidores sobresalientes, disruptores en la industria de medios audiovisuales y opción de consumo atractiva para el público. 
Los servicios de streaming como Netflix, objeto de estudio de esta investigación, han contribuido en la creación de nuevos estilos de consumo, creando audiencias fanáticas y adictas (Murolo y Aon, 2018) que encuentran en este tipo de opción, la satisfacción de necesidades de consumo diversas.

El principal objetivo de esta investigación es describir y comprender el tipo de experiencias de engagement que los jóvenes universitarios tienen con Netflix. De manera específica, en este documento se profundizará en las experiencias educativas que los estudiantes manifiestan tener con esta plataforma. El alcance de este artículo radica en dos aspectos: desde el punto de vista teórico, el engagement emerge como un concepto que ha contribuido de manera importante a entender mejor aquellas experiencias de consumo que aportan más valor a los usuarios de medios digitales, sobre todo tomando en cuenta que los jóvenes son fuertes usuarios de este tipo de medios.

Estas experiencias influyen en su manera de consumir no solo para propósitos de entretenimiento e informativos, sino también para efectos educativos. La complejidad y diversidad de los contenidos de Netflix, otorga posibilidades de consumo variadas; por lo que, desde la perspectiva práctica, resulta de relevancia que la comunidad educativa conozca el tipo de satisfactores que los estudiantes encuentran en este tipo de consumo. Esta investigación se encuentra en una fase exploratoria de conocimiento de un fenómeno social.

\section{REVISIÓN DE LA LITERATURA. CONSUMO DE NETFLIX}

En la era convergente, nuevos agentes han entrado a competir con las empresas tradicionales de medios de comunicación. Algunos de los más relevantes como Google, Amazon, Facebook y Apple, han jugado un rol importante en la transformación de la industria, pues sin ser propiamente medios de comunicación, se han convertido en intermediarios en la provisión de productos audiovisuales (García-Leiva, 2017). También surgieron modelos innovadores de distribución y exhibición de contenidos -como los servicios de video de Internet bajo demanda (VoD) y over the top (OTT)-, por su forma de transmisión a través de la red; es decir, contenido que circula libremente sin que exista control de un operador, a diferencia de los medios tradicionales.

Estos modelos han modificado las lógicas tradicionales de producción de contenido. Lotz (2014) llama a estos fenómenos post-network era, haciendo referencia a la transición de lo lineal o analógico a lo digital, y de las plataformas únicas y diferenciadas, a las multiplataforma. En este ecosistema de medios, el valor del consumo se concentra en el público, más que en el anunciante. Hoy se vive «en» y no «con» los medios digitales; esto implica una orientación distinta del público respecto del flujo y acceso a los contenidos, la propiedad los mismos y la ubicuidad del consumo (Deuze, 2012). No es coincidencia, pues, que las «affordances» de estas formas de consumo sean, justamente, parte integral de la vida cotidiana.

Lo que actualmente conocemos como «Netflix», dista mucho de lo que fue en sus orígenes, en 1997: una compañía que nació con el sueño de Reed Hastings, su co-fundador y actual CEO, que al recibir una multa por la entrega tardía en Blockbuster, soñó con una idea en la que «se pudieran tener DVD's en renta, para tener[los] el mayor tiempo posible sin pagar multas» (Pozzi, 2016; Keating, 2012). El nombre de Netflix es una contracción de 
net (Internet) y de flix, una de las denominaciones de filme en el argot norteamericano. El modelo de negocio, previo a la digitalización, era un servicio de suscripción mensual para acceder a un número ilimitado de DVD's que se enviaban por correo tradicional a las casas. Ahí fue cuando la compañía empezó a ser una competencia real para el mercado de la renta de DVD's.

Tomando en cuenta sus inicios, Netflix insertó en el escenario de medios de ese momento, un modelo de negocio novedoso, que eventualmente también se sirvió del conocimiento de inteligencia artificial de Hastings, permitiéndole crear los primeros algoritmos de lo que sería el soporte tecnológico de su plataforma. Para León-Frías (2021), Netflix se crea en un momento en donde el alquiler y la venta de videos analógicos apuntaba ya una crisis que anticiparía su virtual desaparición posterior; antes del surgimiento de Netflix se había incrementado el video digital bajo la forma de DVD. A esto habría que añadir que también la digitalización de los medios iniciaba su ruta de crecimiento.

En el año 2000 empezó a usarse la tecnología de big data analytics para recomendar contenido a los usuarios del servicio (Cook, 2014). Para 2004, cuando Blockbuster se disponía a lanzar su plataforma en línea de renta de DVD's -al igual que Walmart con su servicio RedBox y Amazon con su servicio LoveFilm-, Netflix ya contaba con casi 2 millones de suscriptores en Estados Unidos, único país donde operaba (Keating, 2012), y ya en las entrañas de la compañía se gestaba la tecnología streaming, a la que migró en 2007.

En 2010, Netflix se internacionalizó: estaba disponible en Canadá, Latinoamérica, el Caribe y Europa. A México llegó en 2011. Otro de sus grandes hitos es el lanzamiento de su producción original, que se hizo posible en el año 2013, con la serie de House of Cards (Block, 2013). Ya en 2011, Netflix había creado su unidad de producción con esta serie que marcó un hito, el rumbo y la evolución de la plataforma streaming.

Como productor original, Netflix se diferencia de otros ofertantes de contenido, como los canales de cable de dos maneras claras:

1) Su oferta apunta al espacio informático.

2) Opta también por transmitir series que otras compañías habían dejado de emitir, descubriendo con ello una potencialidad comercial imprevista (León-Frías, 2021). Esta estrategia, así como su cartera de contenido fílmico reciente y clásico, empiezan a constituir fuentes de interés y engagement para los usuarios.

En 2011, la compañía comenzó a adquirir contenido original para distribuir en su plataforma, que hasta ese momento solo había optado por distribuir contenido cedido por terceros; migrando, eventualmente, a distintos modelos de producción de contenido original (Hidalgo, 2020).

En la actualidad, de acuerdo con Cook (2020), este servicio tiene el dominio del mercado de streaming de video con 193 millones de suscriptores, en los 190 países en los que tiene presencia (Netflix, 2020): 31 millones de ellos están en Latinoamérica (Statista, 2020). Esta región le reportó ganancias por 741 millones de dólares en el año 2019 (Spangler, 2019).

El streaming se ha vuelto un medio popular para acceder a productos audiovisuales. Los mexicanos expresan disposición e interés por consumir contenidos audiovisuales en la red, lo cual puede contemplar tanto opciones OTT, como redes sociales o YouTube. Para 
el usuario mexicano, la principal razón para tener contenido de OTT es pasar tiempo con familia y amigos, o bien para entretenimiento individual (IFT, 2019).

Netflix ha logrado, pues, un alcance de gran escala, no solo relacionado con la captación y fidelidad de mercado a nivel mundial, sino con el impacto que genera en la industria de medios de comunicación, en específico en la de la televisión y el cine. La plataforma es, al día de hoy, uno de los más importantes distribuidores y exhibidores de contenido audiovisual en el mercado internacional (Adhikari et al., 2015) y uno de los sitios con mayor tráfico en el mundo (O'Malley, 2016), junto con YouTube.

Al año 2020, Latinoamérica representa el $20 \%$ de suscriptores de Netflix, estando el 10\% de ellos en Brasil y México. Este alcance se explica de varias maneras: la penetración de Internet en el país, los hábitos de consumo y la oferta de contenido. Ese crecimiento aseguró, sin duda, más visibilidad para sus contenidos y prestigio de marca, aunado a la apuesta por la producción regional, apuesta muy clara para la compañía. De acuerdo con estudios prospectivos de PriceWaterhouseCoopers (2019-2024), para 2022, México superará a Brasil en el mercado total de usuarios de plataformas de streaming en Latinoamérica, por el potencial que tiene el mercado del contenido en esta región. Entre 2019 y 2024, se estima que la industria de entretenimiento y medios en México, obtendrá un crecimiento anual compuesto de $2.27 \%$, y el sector de las OTT podría generar ingresos por 881 millones de dólares al cierre del año 2020: un incremento de $26 \%$ respecto del año 2019. Este crecimiento estará ampliamente influido por los cambios de hábitos a raíz de la pandemia del coronavirus.

La compañía, que se nombra a sí misma como televisión por Internet, es uno de los mejores ejemplos de disruptores en la industria. Jenner (2016) se refiere a este servicio como un ejemplo de televisión III (que usa formas multiplataforma de distribución de contenido y estrategias determinadas de branding, y de programación dirigida hacia nichos específicos), que ha evolucionado a un estado de televisión IV (una televisión fluida donde se cruzan estructuras industriales y tecnológicas, diversas formas de accesibilidad para los usuarios, patrones de consumo particulares y estrategias de branding), a partir de aprovechar un ecosistema mediático de cambios en términos tecnológicos y de comportamientos del consumidor.

Según Medina, Herrero y Urgellés (2017), con información de Small (2012) y Vonderau (2015), el valor de la propuesta de Netflix radica en tres ejes principales:

1) Amplio catálogo de contenido para que los usuarios puedan elegir.

2) Útil e innovador motor de recomendaciones.

3) Variedad de estrategias de cobro.

A su vez, Lobato (2015) añade que la estrategia de internacionalización y a la vez de producción de contenidos originales, ha sido primordial para su penetración en los mercados a los que ha llegado. Small (2012) lo describe en términos de la construcción de su estrategia, basada en la producción original, el contenido de terceros, el contenido licenciado a estudios o cadenas extranjeras para completar el catálogo, y el contenido licenciado a empresas nacionales; asimismo, la accesibilidad de su precio en los mercados en los que se sitúa, y el valor diferenciador de una interfaz sencilla y un sistema de recomendación efectivo (Strangelove, 2015), aseguran que la tercera parte del contenido en la plataforma es el resultado del software de recomendación que conoce: lo que los usuarios consumen; cuándo lo miran; si ven solo 
una parte o el contenido completo; cuándo regresan, y cuándo vuelven a interactuar con un contenido. Todos estos elementos posibilitan a los suscriptores vivir la experiencia Netflix.

\section{«ENGAGEMENT»CON MEDIOS DIGITALES}

¿Cómo se genera la conexión de las personas con los medios que consumen?, ¿por qué, existiendo variadas opciones de medios y contenidos, algunos logran mayor atención?, ¿por qué las audiencias eligen un contenido sobre otro?, ¿por qué el público cambia su comportamiento de consumo con regularidad?, ¿por qué las personas se relacionan con otras, a partir de los contenidos que consumen? Estas son algunas preguntas que pueden tener al engagement como concepto relevante, aglutinador y como fenómeno de interés en diferentes campos en los que se desea entender las emociones, los pensamientos y las acciones de las personas. Como uno de los primeros antecedentes de este concepto, Csikszentmihalyi (1975, p. 36) hablaba de la existencia de «una sensación holística que las personas sienten cuando actúan con total implicación», que también llamó flow experience, o estado de total involucramiento en una actividad que requiere de atención y concentración. Este psicólogo desarrolló estudios sobre terapia ocupacional, motivación intrínseca, ansiedad y aburrimiento, entre otros.

A partir de su investigación, buscaba contestar a las preguntas: ¿qué hace involucrarse a una persona con una actividad? y ¿qué tipo de compensaciones están detrás de ese involucramiento? Desde entonces se prospecta ya un constructo psicológico para entender las experiencias subjetivas del compromiso con una actividad, pues la implicación con una actividad se relaciona con estados cognitivos, creencias de identificación psicológicas y emociones (Kanungo, 1982).

En la actualidad, los usos más comunes de este concepto surgen en el contexto del empleo de las redes sociales, el ámbito laboral, la responsabilidad social, el compromiso cívico, el capital social y el ámbito educativo (Taylor y Kent, 2014; Fredericks et al., 2004). Se ha demostrado que el engagement con temas políticos, por ejemplo, repercute en la generación de capital social (Gil de Zuñiga et al., 2012) y en el fortalecimiento de las instituciones propias del sistema democrático, debido a que en la actualidad los medios digitales permiten la participación y el activismo digital (Givskov y Trenz, 2014). En el área del entretenimiento, ha sido un concepto relevante para analizar los factores que crean engagement con los videojuegos. Brown (2011) ha buscado desarrollar modelos para conocer por qué las personas, a partir del concepto de engagement, asisten a espectáculos en vivo. Como puede observarse, este concepto posee mucho potencial y ayuda a entender cómo las personas actúan de maneras similares en escenarios distintos, lo cual agrega complejidad a este concepto.

En el ámbito de la comunicación, los modelos de engagement han sido ampliamente discutidos desde la academia y la industria. En los últimos años, la importancia de analizar el comportamiento de los diferentes públicos ha ido en aumento, acrecentando la relevancia del concepto de engagement de la audiencia (Ferrer-Conill y Tandoc, 2018). El engagement es un constructo que descifra las dimensiones psicológicas y de comportamiento social que explican la vinculación individual y social experimentada al consumir contenidos de comunicación, o al emplear alguna plataforma digital específica (Johntson y Taylor, 2018).

Existe también la conceptualización del engagement platform, que supone la relevancia de las interfaces como facilitadoras de la construcción del vínculo desarrollado por un usuario 
hacia una marca, producto o servicio (Brodie, Ilić, Jurić y Hollebeek, 2013; Harris y Dennis, 2011). Las plataformas que facilitan la interacción de los usuarios suelen alcanzar mayores niveles de engagement (Van Weezel y Benavides, 2010). En esa misma línea, Lin y Parsons (2018) argumentan que el engagement puede ser útil para mantener conectados a los usuarios a una plataforma o contenido cuando están realizando multitareas.

En el caso de esta investigación, interesa conocer la diversidad de experiencias psicológicas, emocionales y de comportamiento, que una persona percibe al consumir una plataforma como Netflix. Para estos efectos, el modelo seleccionado es el de David Mersey, Malthouse y Calder (2010). A través de este modelo se pretende también conocer más sobre las creencias, los pensamientos y las ideas que los estudiantes tienen sobre su consumo de Netflix, a través de una colección de experiencias que aluden a las creencias e ideas que los consumidores manifiestan tener al consumir contenidos, y cómo eso que consumen les aporta sentido en diversos aspectos de su vida.

Para David Mersey, Calder y Malthouse (2010), las experiencias de los usuarios poseen una dimensión personal y una social-interactiva. La personal hace referencia al conjunto de manifestaciones que suelen tener con medios tradicionales; por otro lado, la parte social-interactiva es más específica para el consumo de medios en Internet, e incluso este conjunto de experiencias se comparten con lo que un usuario experimenta consumiendo redes sociales. En general, a través del consumo de medios de comunicación, las personas buscan estimulación, inspiración, interacción con otros a propósito del contenido, la afirmación de autoestima y las sensaciones intrínsecas de gozo o satisfacción. En las experiencias de engagement social-interactivo siempre están incluidas las personales. En complementariedad, el compromiso social-interactivo está motivado por la relevancia social, y una experiencia de compromiso y de comunidad mucho más amplia, por eso se valoran en estas dimensiones aspectos relativos a la participación con otros y la socialización. El sentido de utilidad y de gozo intrínseco, también están presentes en estas vivencias de implicación.

Estas experiencias, a su vez, involucran dimensiones diversas de engagement, mediante las cuales las personas expresan lo que entienden sobre sus comportamientos, razonamientos y sentimientos en relación con su consumo de medios de comunicación. Las dimensiones comprenden: experiencias de estimulación e inspiración, de facilitación social, de temporalidad, de autoestima y conciencia cívica, de entretenimiento intrínseco, de utilidad, de participación y socialización, y de sentido de comunidad. De acuerdo con este modelo, algunas dimensiones abarcan experiencias de engagement individual y otras experiencias sociales e interactivas. A partir de las 37 experiencias concretas, se genera el cuestionario de medición seleccionado para este trabajo de investigación: 
Figura 1. Modelo de uengagement»

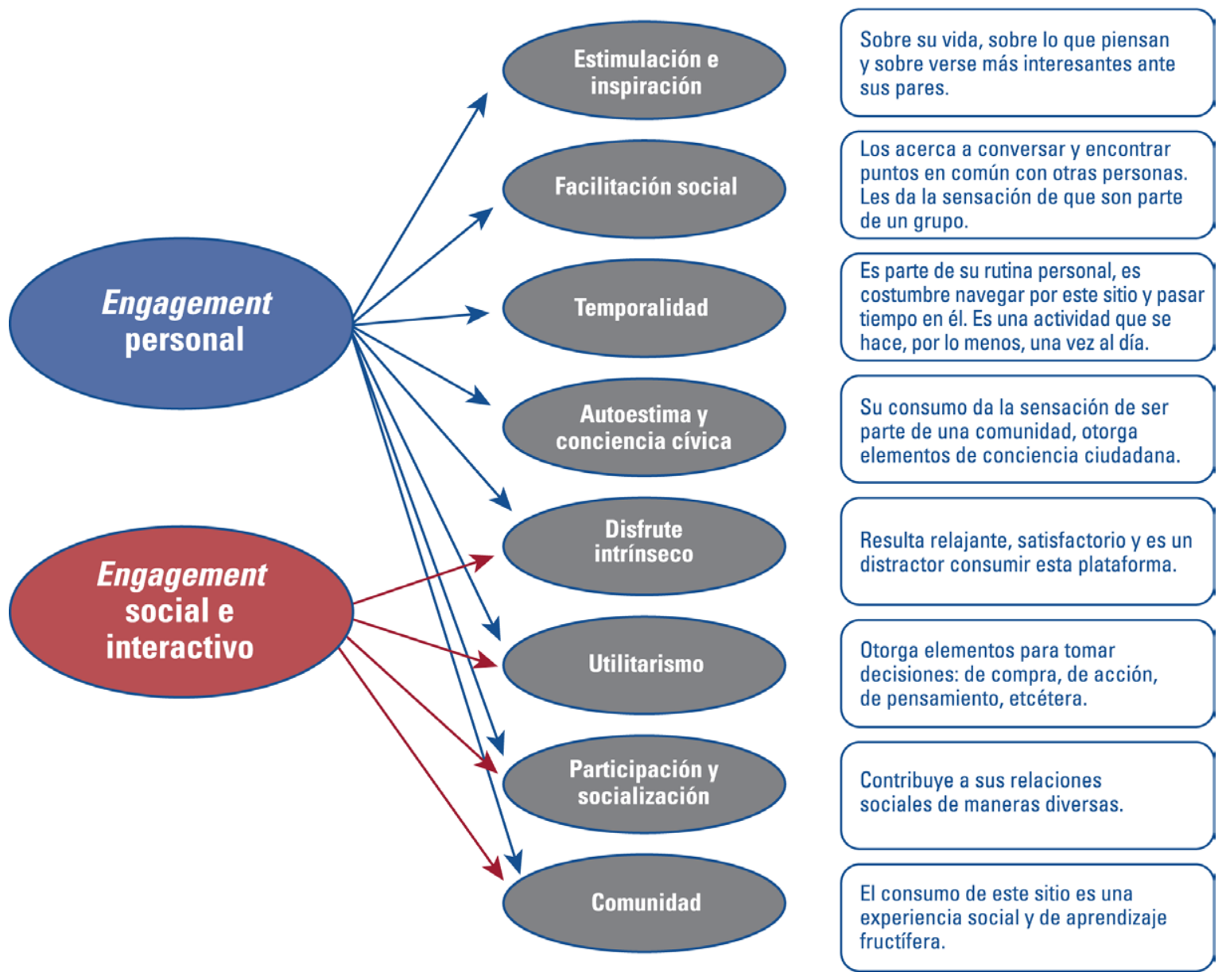

Fuente: Elaboración a partir del modelo de David Mersey, Malthouse y Calder, 2010.

\section{MÉTODO}

El propósito de este artículo es conocer los indicadores de engagement con la plataforma de video bajo demanda (streaming) Netflix, así como las características de consumo de los jóvenes mexicanos universitarios de 18 a 26 años, tomando como referencia de medición el modelo de engagement con medios digitales de David Mersey, Malthouse y Calder (2010). Con este objetivo se pretende conocer -con mayor profundidad-creencias, pensamientos e ideas que los sujetos de investigación tienen sobre sus experiencias de engagement con esta plataforma, así como las motivaciones profundas derivadas de esos indicadores de engagement más recurrentes en su consumo -desde la voz de los jóvenes universitarios-, particularizando en las experiencias educativas que manifiestan experimentar con la plataforma.

Aunado a lo anterior, esta investigación pretende los siguientes objetivos específicos:

a. Probar la efectividad del empleo del paradigma de engagement para medir el consumo de plataformas audiovisuales como Netflix. 
b. Conocer la percepción de las audiencias sobre sus usos y costumbres de consumo, profundizando en los criterios de engagement propuestos por David Mersey, Calder y Malthouse, y detectando categorías de consumo a partir de la voz de los estudiantes.

Desde la perspectiva empírica, esta investigación gira alrededor de una pregunta principal: ¿cuáles son las experiencias de consumo audiovisual de las audiencias universitarias mexicanas, con plataformas audiovisuales de streaming?

Se trata de una investigación mixta secuencial, porque se profundizará en resultados obtenidos mediante varios métodos de recolección de datos cuantitativos y cualitativos, empleando uno posteriormente al anterior para tener una mejor comprensión del objeto de estudio. Es no experimental: no existe intención de manipular deliberadamente las variables a investigar, sino observar un fenómeno tal y como se lleva a cabo en su entorno natural. Es transeccional porque los datos se recolectarán en un solo momento (Fernández, Baptista y Hernández, 2014).

El alcance descriptivo de esta investigación busca reconocer las experiencias de engagement experimentadas en el consumo de Netflix, más allá de la mera descripción de variables, lo mismo que a contestar por qué y en qué condiciones se manifiestan esas experiencias en Netflix. Desde esta perspectiva, la combinación de dos perspectivas de investigación ayuda a brindarle mayor profundidad y alcance. Steiner y Xu (2018) resaltan la necesidad de realizar estudios cualitativos para conocer las motivaciones de los usuarios en el consumo de plataformas audiovisuales, enfatizando en las experiencias de carácter educativo.

El instrumento cuantitativo seleccionado para la recolección de datos fue un cuestionario electrónico y auto administrado, con preguntas cerradas de respuesta única y escalamiento estimativo tipo Likert. Los ítems se adaptaron a partir del modelo original de David Mersey, Malthouse y Calder (2010), contextualizándolos a la utilización de Netflix con una escala estimativa en que los usuarios pudieran señalar el nivel de acuerdo o desacuerdo que tienen sobre las 37 experiencias de engagement identificadas en el mismo. También se añadieron otras preguntas que permitieran contar con información descriptiva sobre la muestra seleccionada.

La muestra fue por conveniencia, se utilizó una probabilidad de acierto (p) de 0.5 para determinar el tamaño de la muestra, con un nivel de confianza (z) de $95 \%=1.96 \%$ y un margen de error (e) de 5\%. Lo anterior dio como resultado una muestra (n) de 384 jóvenes, y se logró llegar a una muestra final de 630 encuestas de estudiantes de universidades públicas y privadas de zonas urbanas de México, y un perfil variado. Entre la muestra, los participantes estudiaban 41 tipos de carreras universitarias distintas. 39\% fueron hombres y $61 \%$ mujeres.

Al ser México un país grande, era importante asegurar la representatividad de alumnos de las diversas regiones. Existen algunas características del contexto universitario mexicano que hicieron esto posible (Anuies, 2019):

a. El 65\% de estudiantes universitarios en universidades de la Ciudad de México, el estado de México y los estados de Puebla, Nuevo León y Jalisco, son foráneos. Por otro lado, los estados que menos reciben estudiantes foráneos son Oaxaca, Guerrero y Zacatecas.

b. 1 de cada 6 estudiantes de cualquier aula universitaria del país, son estudiantes foráneos (que llegan de poblaciones cercanas que no tienen universidades, o bien, ellos desean estudiar en una universidad lejos de su lugar de origen). 
c. La dinámica de migración estudiantil se determina por la cercanía, pues a pesar de que el $70 \%$ de los estados reciben al menos a un estudiante de cada entidad, los grupos más grandes suelen ser de los estados colindantes.

En algunos resultados descriptivos de la muestra, se pueden identificar los dispositivos de uso.

\section{Gráfico 1. Dispositivos con los que cuentan}

\section{Dispositivos de uso}

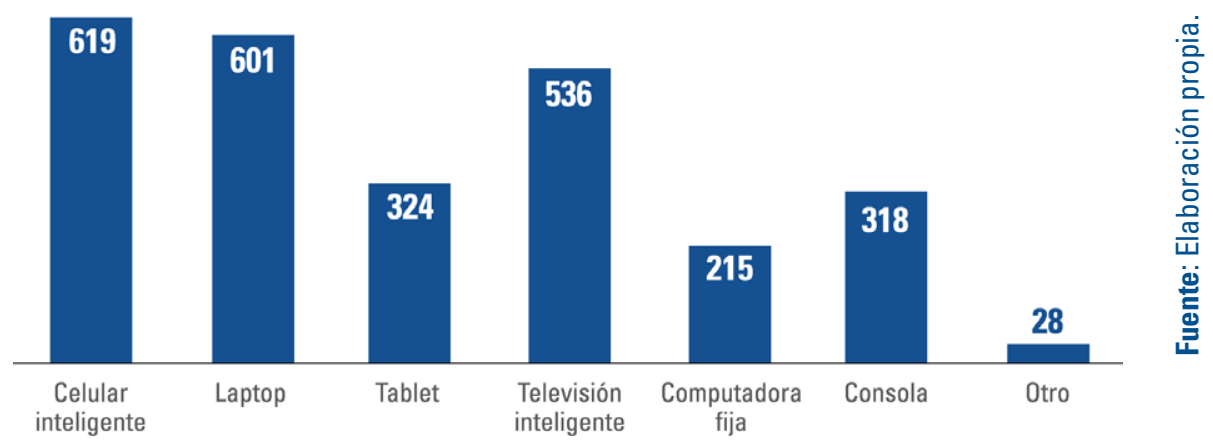

El empleo del móvil y la computadora personal tienen el dominio de utilización. Por otro lado, cabe resaltar la presencia cada vez más relevante de la consola como dispositivo con multifunciones, en sintonía con información de IAB y Kantar (2020) que reportó que, en el primer año de pandemia, la televisión inteligente y las consolas fueron los dispositivos de mayor crecimiento en el año; mientras que el teléfono inteligente y la computadora personal se mantuvieron en un nivel semejante al del año anterior del mismo estudio. El internauta mexicano promedio tiene 4.9 dispositivos, de los cuales emplea 3.6 para conectarse a Internet (IAB y Kantar, 2020). Cabe señalar que un filtro para realizar esta encuesta es que se tuviera una cuenta de Netflix: el 100\% de la muestra tiene acceso a una cuenta individual o familiar de esta plataforma. De acuerdo con la encuesta, el promedio de tiempo que los estudiantes pasan en Netflix es de 164 minutos entre semana y de 212 minutos en fin de semana.

\section{Gráfico 2. Dispositivos usados para ver plataformas de streaming} ¿Dónde ven Netflix?

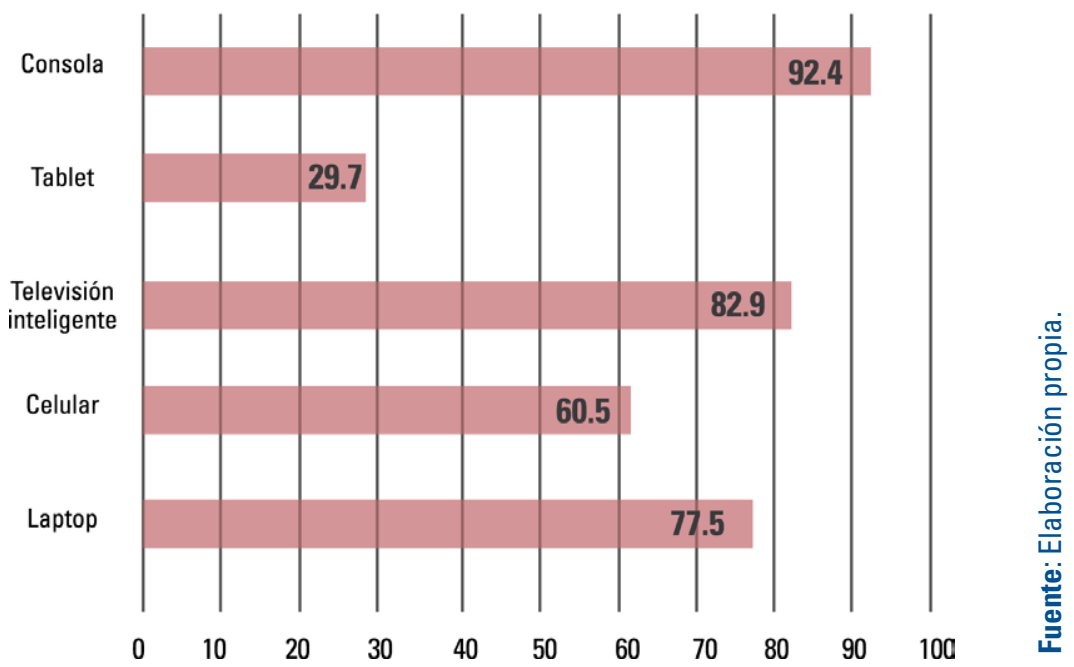


Respecto de la disposición de pago, es evidente la dependencia económica de los estudiantes universitarios mexicanos pues, entre la muestra, tan solo el 8.2 pagan su cuenta de Netflix de manera individual y al 91.8 restante se la paga alguien más (generalmente algún miembro de su familia). Este fenómeno confirma también la práctica generalizada de compartir cuentas de Netflix, que de acuerdo con Sweney (2021) con información de Cordcutting, son alrededor de 40 millones las cuentas «prestadas» de usuarios principales; por lo que la compañía, en la búsqueda por generar más beneficios económicos, se plantea introducir ciertos límites para forzar nuevas altas entre usuarios que comparten sus cuentas. Por lo menos, en la muestra se corrobora que los usuarios más jóvenes en México no pagan su propio servicio; no obstante, probablemente sean quienes más horas pasen en la plataforma, pues en el estudio, los estudiantes afirman ver 1.86 hrs. al día de Netflix entre semana y 2.26 en fin de semana.

En cuanto al análisis central del estudio cuantitativo, se corrieron dos tipos de análisis, uno de fiabilidad de Cronbanch que reportó un resultado de 0.922 , lo cuál corrobora la pertinencia del modelo para medir experiencias de consumo con Netflix. El segundo fue un análisis factorial, que es una técnica de reducción de datos que sirve para encontrar grupos homogéneos de variables a partir de un conjunto usualmente grande de variables, lo cual sirve para encontrar y establecer una estructura interna, generando factores a partir de un conjunto de constructos.

En cuanto a los atributos de engagement, se corrobora que los jóvenes universitarios relacionan el consumo de Netflix con experiencias de descanso y satisfacción personal, así como con experiencias socializadoras; y también aparecen experiencias de inspiración y estimulación. Se detecta que las experiencias de consumo con Netflix tienen elementos muy variados, y se identifican los aspectos relacionados con la utilización de la plataforma, con las rutinas y los hábitos arraigados.

\section{Tabla 1. Atributos más altos de engagement con Netflix de acuerdo al modelo de referencias}

\begin{tabular}{|l|l|l|}
\hline \multicolumn{1}{|c|}{ Atributo } & 4.20 & 1.807 \\
\hline El contenido de Netflix inspira aspectos de mi propia vida. & 4.62 & 1.768 \\
\hline El contenido de Netflix me hace pensar de nuevas maneras. & 5.15 & 1.557 \\
\hline El contenido de Netflix me estimula a pensar de otros temas. & 1.807 \\
\hline El contenido de Netflix inspira aspectos de mi propia vida. & 4.20 & 1.806 \\
\hline $\begin{array}{l}\text { El contenido que he visto en Netflix me ha hecho pensar con } \\
\text { profundidad. }\end{array}$ & 4.66 & 1.703 \\
\hline $\begin{array}{l}\text { Me he descubierto hablando sobre Netflix o sobre contenido } \\
\text { de Netflix en una conversación con otras personas. }\end{array}$ & 5.57 & 1.867 \\
\hline $\begin{array}{l}\text { Ver Netflix me da herramientas para tener conversación con } \\
\text { otras personas. }\end{array}$ & 4.78 & 1.832 \\
\hline $\begin{array}{l}\text { Creo que los usuarios de Netflix podemos aprender del } \\
\text { contenido que encontramos ahí. }\end{array}$ & 4.59 & 1.835 \\
\hline $\begin{array}{l}\text { Me intereso por temas a partir de que vi algún contenido de } \\
\text { Netflix. }\end{array}$ & 4.45 & \\
\hline
\end{tabular}




\begin{tabular}{|l|l|l|}
\hline El uso de Netflix me da pie a socializar sobre diversos temas. & 4.45 & 1.857 \\
\hline El contenido de Netflix me da temas de conversación. & 5.35 & 1.666 \\
\hline $\begin{array}{l}\text { En Netflix se puede encontrar contenido para mejorar en } \\
\text { distintos aspectos. }\end{array}$ & 4.20 & 2.057 \\
\hline $\begin{array}{l}\text { En alguna situación he usado contenido visto en Netflix para } \\
\text { argumentar o discutir sobre un tema. }\end{array}$ & 4.47 & 1.731 \\
\hline $\begin{array}{l}\text { Me gusta ver Netflix cuando estoy tomando un descanso o } \\
\text { comiendo. }\end{array}$ & 5.43 & 2.102 \\
\hline $\begin{array}{l}\text { Ver Netflix se ha convertido en parte de mi rutina. } \\
\text { Ver Netflix me relaja. }\end{array}$ & 4.22 & 1.593 \\
\hline $\begin{array}{l}\text { Uso Netflix como parte importante del contenido de } \\
\text { entretenimiento que consumo. }\end{array}$ & 5.32 & 1.806 \\
\hline $\begin{array}{l}\text { Ver Netflix mejora mi estado de ánimo, me hace sentir más } \\
\text { contento. }\end{array}$ & 4.96 & 1.892 \\
\hline \begin{tabular}{l} 
Ver Netflix me provoca satisfacción. \\
\hline $\begin{array}{l}\text { He encontrado en Netflix contenido que refleja mis valores } \\
\text { personales. }\end{array}$
\end{tabular} & 4.26 & 1.794 \\
\hline
\end{tabular}

Fuente: Elaboración propia. La escala estimativa de elección es entre 1-7.

De acuerdo con este análisis de medias, se identifican algunas experiencias relacionadas con satisfacción de necesidades educativas. Los alumnos universitarios expresan que la plataforma ofrece contenidos que los motivan a pensar en diferentes tópicos, que se interesan en nuevos temas a partir del consumo de Netflix y, sobre todo, que su contenido otorga elementos de aprendizaje, más allá del entretenimiento.

En general, las experiencias con una media más alta, permiten identificar que los estudiantes universitarios relacionan el consumo de Netflix con la autonomía y personalización; la plataforma y su contenido mejoran, transforman y extienden sus experiencias de consumo a momentos de socialización y de consumo socializado, tanto en redes sociales como de manera presencial. También aprecian la diversidad de contenidos y temáticas; la plataforma les abre opciones de consumo de contenidos que no tenían antes a su disposición. Algunas vivencias negativas presentes en el consumo de esta opción de streaming, pueden relacionarse con lo que se conoce como la «fatiga de decisión» o la «paradoja de la elección», en términos de Barry Schwartz, que para efectos de la utilización de una OTT, puede traducirse en saturación mental entre tantas opciones. Esta experiencia puede convertirse en una desventaja, pues podría afectar de manera indirecta las horas de reproducción y, por tanto, la fidelización de los clientes (Neira, 2020). Los usuarios de Netflix reconocen la existencia de procesos algorítmicos y los aceptan, e incluso los entienden en función de la oferta presentada.

Por otro lado, se realizó un estudio cualitativo que consistió en la realización de 8 focus groups que, en total, suponen la participación de 46 estudiantes. Soler (1990) considera al focus group como uno de los métodos más eficaces para proporcionar conocimiento y comprensión profunda sobre las necesidades, motivaciones, actitudes, los deseos y comportamientos del 
consumidor, ya que permite establecer los sistemas de valor, atributos y significados -conscientes e inconscientes- asociados al consumo de algún producto o marca. Asimismo, frente al hecho de que no existen muchos estudios previos sobre experiencias de engagement, particularmente con plataformas audiovisuales, se optó por emplear la técnica cualitativa del focus group para obtener información preliminar sobre estos tópicos. Esta técnica permitió recolectar información de los participantes sobre sus experiencias de engagement en sus propias palabras. Emplear técnicas cualitativas es útil en el estudio de temas que están en una etapa exploratoria (Rubenking y Sandoval, 2018). Además, la experiencia y práctica de esta técnica posibilitó la obtención de insights sobre las prácticas de consumo con plataformas VoD.

Los focus group se realizaron de manera virtual, dadas las restricciones de la pandemia, pues se llevaron a cabo entre junio y julio de 2020. En este sentido, vale la pena considerar que la realización cualitativa en línea, previa a la pandemia, es cada vez más una forma común de obtención de información, y en los grupos focales virtuales también es posible generar interacción valiosa (Sweet, 2001; Stewart y Shamdasani, 2016).

Entre las características del perfil de los participantes, pueden resaltarse los siguientes elementos:

- Todos los participantes tienen acceso a más de una suscripción, en su mayoría, además de Netflix, las más mencionadas fueron: Amazon Prime, HBO y Apple TV. Además de las plataformas anteriores, también: Klic, YouTube Red, XBox Game Pass, Roku. La mayoría tenía expectativa de la llegada de Disney +, que arribó al país 3 meses después: el 17 de noviembre de 2020.

- Entre los dispositivos más empleados: celular y computadora personal (100\%), tablet $(46 \%)$ y una televisión inteligente $(77 \%)$ que suele estar en el espacio familiar.

- Tienen utilizando Netflix, un promedio de 5 años.

- Un 30\% además de estudiar, trabajan y 70\% solo estudian.

- Los participantes son estudiantes de carreras como: comunicación, pedagogía, mercadotecnia, economía, ingeniería e innovación, ciencias políticas y derecho.

- Un $40 \%$ fueron hombres y un $60 \%$, mujeres.

- Viven en las ciudades de: Guadalajara, Zapopan, Ciudad de México, León, Aguascalientes y Monterrey.

El contenido se exploró mediante un método lógico-semántico (Munita, 2016), un enfoque de categorización que combina análisis temáticos, estructurales y de frecuencia.

Después de leer y analizar las transcripciones de los focus group, se identificaron unidades de significado que fueron agrupadas en categorías de análisis que se interrelacionaron, se integraron y refinaron en un mayor nivel de abstracción (Creswell, 2002) y, a su vez, también se buscó concomitancia temática con experiencias de engagement del modelo de referencia. Para efectos de este artículo solo se describirán las experiencias de orden educativo identificadas, que son dos: 


\section{Conocimiento y comprensión del contexto}

Netflix visibiliza temas y muestra contenidos de cuestiones coyunturales. Sus contenidos facilitan conocer: otras perspectivas sobre hechos y puntos de vista; referencias culturales ajenas; así como sensibilizar sobre asuntos con los que en principio pudiera haber desacuerdo, o bien, desconocimiento; lo mismo que reafirmar convicciones. La oferta de contenidos universales facilita adentrarse en otros entornos y viajar sin salir de casa.

- «Netflix me ha influido mucho. Una serie me hizo hacer un viaje; fui más por la serie que por otra cosa, y también casi me hago doctora por otra serie. Me gusta hablar y comentar con alguien y leer otras cosas» (Lorea, 21).

- «Lo que define a la plataforma es la variedad de contenido y de temas en esos contenidos» (Pepe, 25).

- «Netflix significa una oportunidad de conocer un poco más porque, si te fijas, ya no solo te dan esa apertura de contenidos de México y EUA, ya ves contenido latinoamericano, ruso, de todos lados $[\ldots]$, es como una ventaja de conocer nuevos contenidos. Para mí significa una gran ventaja» (Luis, 23).

- «Relaciono el consumo de Netflix con diversidad, contenido cultural. Yo escribo y lo uso también para la búsqueda de historias» (Marisol, 22).

En el focus group, fue muy llamativo advertir que Netflix es una plataforma que también se utiliza para informarse; consumir su contenido motiva a buscar información sobre otros temas; y que al ver contenido internacional, este ayudaba a entender el contexto de otras personas.

\section{Juicio y reflexión}

Comprender asuntos complicados, profundizar sobre ideas y conceptos, genera reflexión y conversación a los estudiantes sobre temas que se desconocían, motivándoles a ser analíticos sobre historia, política y cuestiones sociales; los contenidos les retan intelectualmente y fomentan la imaginación, reforzando los propios intereses.

- «Por la cantidad de contenido a la que estamos expuestos en Netflix, hay mucha variedad de temas, $[\ldots]$ es una oportunidad de tener conversaciones sobre temas nuevos y relevantes, conversaciones que no estábamos teniendo» (Ireri, 24).

- «Otra experiencia es que a través de las series se tocan diferentes temas -aunque sea una serie-, también como que te dan cierta perspectiva de las cosas; te hacen pensar muchísimo» (Ana Paula, 20).

- «Estas plataformas también tienen el objetivo de visibilizar ciertos temas. Te lleva a pensar o simpatizar con situaciones, cuestionar actitudes, empatizar [...]» (Michelle, 21).

- «Hay temas controversiales. Te das cuenta de muchas cosas. Te hace reafirmar la manera en que crees ciertas cosas o hasta verlo desde otro punto de vista. Me ha hecho cuestionarme mucho, reafirmar» (Regina, 24).

- «A mí personalmente me gustan los lugares donde están filmadas las películas y series, y ya quiero ahorrar para ir a ese [determinado] pueblo» (Renata, 24).

El contenido de Netflix ayuda a reflexionar y confirmar convicciones personales; es una herramienta educativa, e inclusive, a partir de lo que se ve y analiza en el momento, pueden tomarse notas o comentarlo reflexivamente, porque se le valora como contenido de calidad que aporta valor desde la perspectiva del aprendizaje. 


\section{DISCUSIÓN Y CONCLUSIONES}

Las audiencias se conectan de diferentes maneras con los medios y contenidos de comunicación que consumen; poseen un mayor control sobre la forma en la que viven su consumo de medios de comunicación. Este estudio se aproxima a entender las formas específicas en las que se produce un consumo que, de entrada, se asume activo, conociendo la percepción sobre el engagement en el uso de plataformas como Netflix. Este artículo gira en torno, específicamente, sobre cómo los estudiantes universitarios generan experiencias de consumo educativas con Netflix. Esto quiere decir que las motivaciones detrás del consumo de esta plataforma, no únicamente están relacionadas con satisfactores de entretenimiento.

Es posible utilizar modelos de engagement para reconocer el vínculo de las personas con las plataformas y con los contenidos que consumen. Autores como Bergillos (2017) consideran que, respecto del consumo digital, se prevé que las audiencias tenderán a ser más conscientes y conocedoras de su forma de consumo, reconociendo que les gusta algún contenido y que, por tanto, merece su tiempo y atención.

Este análisis exploratorio ha permitido identificar que los contenidos visionados en la plataforma de Netflix, estimulan cognitivamente a los estudiantes; aunque para ellos esta estimulación tiene dos aristas: ayuda a entender mejor el contexto y determinados fenómenos sociales, o bien, es usado para propósitos educativos.

El modelo de David Mersey, Calder y Malthouse ayuda a comprender las experiencias de engagement al consumir Netflix, y cómo ese consumo trasciende las experiencias de relajación y conecta a los estudiantes universitarios con otras personas, con su contexto social, con sus pensamientos, con su juicio crítico al considerar que su uso resulta útil, pues aprenden de los contenidos que ven.

La edad es un factor relevante para el tipo de consumo, para el pago por plataformas, para el tiempo que se consume y para la conciencia sobre el engagement que se tiene con el consumo; se observa que, según la muestra, entre los estudiantes universitarios, el engagement se manifiesta de diversas maneras.

A partir de la aplicación de este modelo se logra distinguir que el público joven se relaciona de manera particular con la marca Netflix, y estos usuarios experimentan motivaciones diferenciadas y variadas al usar la plataforma. Tomando en cuenta que el modelo de engagement elegido describe una colección más vasta de experiencias, los resultados de esta investigación permiten identificar: experiencias educativas para Netflix; la forma en que su consumo conecta a los jóvenes con otros aspectos de su vida, y la manera en que el consumo de plataformas digitales de suscripción se ha convertido en parte importante de usos y costumbres de ocio e información.

Este análisis responde a la pregunta inicial de investigación, identificando experiencias específicas de consumo con Netflix. Asimismo se encontró: una dedicación de tiempo relevante al consumo de contenido digital; la incorporación de rutinas a sus actividades diarias; la socialización de contenidos que se consumen en plataformas, como temas de conversación con sus pares, y la detonación de su interés por determinados temas.

Con este estudio exploratorio se identifica que Netflix es muy empleado por los jóvenes mexicanos, que estos son un público consumidor de plataformas digitales, que se han adaptado a los efectos de la convergencia y que se encuentran dispuestos a nuevas formas de consumo por lo que ellas les ofrecen. 
El impacto educativo de este estudio radica en el descubrimiento de que los contenidos de entretenimiento influyen en aspectos más profundos de la vida de las personas, en este caso, los estudiantes universitarios. Ellos confían y procuran el consumo de entretenimiento como complemento de sus necesidades de aprendizaje; su conexión con el entretenimiento les conduce a razonamientos más profundos sobre lo que sucede en su vida y en su contexto. Asimismo, los educadores podrían beneficiarse de estas experiencias de engagement para aprovecharlas estratégicamente y emplear los contenidos de plataformas, de manera intencionada, como recurso didáctico; esta decisión despertaría de inicio -muy probablemente-, el interés de los alumnos.

A través de este artículo se patentiza que los medios de comunicación, si bien buscan entretener e informar principalmente, tienen la potencia de impactar educativamente a las generaciones de jóvenes y que los medios de comunicación deben ser aliados de la educación formal como estrategia y recurso didáctico importante.

\section{REFERENCIAS}

Adhikari, V. K.; Guo, Y.; Hao, F.; Hilt, V.; Zhang, Z. L.; Varvello, M. \& Steiner, M. (2015). Measurement study of Netflix, Hulu, and a tale of three CDNs. IEEE/ACM Transactions on Networking, 23(6), 1984-1997.

Albarran, A.; Mierzejewska, B. \& Jung, J. (Eds.). (2018). Handbook of media management and economics. Routledge.

Bergillos, I. (2017). ¿Dos caras de la misma moneda?: una reflexión sobre la relación entre engagement y participación en medios. Comunicación y hombre 14, 121-136.

Block, A. B. (2013). Media execs divided on Netflix's 'House of Cards' model. The Hollywood Reporter. http:// www.hollywoodreporter.com/news/media-execs-dividednetflixshouse-418357

Brodie, R. J.; Ilić, A.; Jurić, B. \& Hollebeek, L. (2013). Consumer engagement in a virtual brand community: An exploratory analysis. Journal of Business Research, 66(1), 105-114. https:// doi.org/10.1016/j. jbusres.2011.07.029

Brown, A. \& Ratzkin, R. (2011). Making sense of audience engagement. The San Francisco Foundation, 1, 78.

Cook, C. (2014). Netflix: A stepping stone in the evolution of television. University of South Florida. http:// dspace.nelson.usf.edu/xmlui/handle/10806/11681

Cook, S. 2020. 50+ Netflix statistics and facts stats that define the company's dominance [2020 version]. Comparitech.

Csikszentmihalyi, M. (Ed.). (1975). Beyond boredom and anxiety: The experience of play in work and games. San Francisco, CA: JosseyBass.

Cruz-Sotelo, S. E.; Benítez, S. O.; Bovea, M. D.; Soto, N. S.; Ávila, H. F. y Salinas, W. E.A. (2013). Hábitos y prácticas de consumo de teléfonos celulares en México y España. Revista Internacional de Contaminación Ambiental, 29, 33-41.

David Mersey, R.; Malthouse, E. C. \& Calder, B. J. (2010). Engagement with online media. Journal of Media Business Studies, 7(2), 39-56. 
Deuze, M. (2012). Media life. Cambridge: PolityPress.

Domínguez, D. y López, R. (2015). Uso de las redes sociales digitales entre los jóvenes universitarios en México. Hacia la construcción de un estado del conocimiento (20042014). Revista de comunicación, 14, 48-69.

Fernández-Beaumont, J. (2000). Digitalización, convergencia, multimedia. Claves del nuevo escenario audiovisual español. Cuenta y razón, 117, 37-46.

Fernández Collado, C.; Baptista Lucio, P. y Hernández Sampieri, R. (2014). Metodología de la Investigación. México: Editorial McGraw Hill.

Ferrer-Conill, R. \& E. C. Tandoc Jr. (2018). The Audience-Oriented Editor. Digital Journalism, 6, 436-453. https:// doi.org/10.1080/21670811.2018.1440972

García-Leiva, M. T. (2017). Desafíos y oportunidades para la diversidad del audiovisual en Internet. Política \& Sociedade. Florianópolis, 16(35), 2017. https: / / doi.org/10.5007/2175$\underline{7984.2017 \mathrm{v} 16 \mathrm{n} 35 \mathrm{p} 132}$

Gil de Zuñiga, H.; Jung, N. \& Valenzuela, S. (2012). Social Media Use for News and Individuals' Social Capital, Civic Engagement and Political Participation. Journal of Computer-Mediated Communication, 17(3), 319-336. https:// doi.org/10.1111/j.1083-6101.2012.01574.x

Givskov, C. \& Trenz, H. J. (2014). Civic engagement through mainstream online newspapers: Possibilities and shortcomings. MedieKultur. Journal of Media and Communication Research, 30(56), 44-60. http:/ / ojs.statsbiblioteket.dk/index.php/mediekultur/article/ view $/ 16969 / 15302$

Jenner, M. (2016). Is this TVIV? On Netflix, TVIII and binge-watching. New media $\mathcal{E}$ society, 18(2), 257-273.

Johnston, K. A. \& Taylor, M. (Eds.). (2018). The handbook of communication engagement. John Wiley \& Sons.

Harris, L. \& Dennis, C. (2011). Engaging customers on Facebook: Challenges for e-retailers. Journal of Consumer Behaviour, 10, 338-346. https:// doi.org/10.1002/cb.375

Hidalgo T. (2020). Netflix como productor audiovisual. Obra Digital, 19, 117-132. https: / doi. org/10.25029/od.2020.272.19

IAB (2020). Estudio de consumo de medios y dispositivos entre internautas mexicanos. https:// d335luupugsy2.cloudfront.net/cms/files / 65507/1600450982ECMyD 2020 CorteGeneralVP.pdf

Instituto Federal de Telecomunicaciones IFT (2019). Encuesta Nacional de Consumo Audiovisual ENCCA, 2019. http://www.ift.org.mx/sites/default/files/contenidogeneral/mediosy-contenidos-audiovisuales/01encca2019vp.pdf

Kantar Media (2020). Cómo han cambiado los hábitos de consumo de TV durante el COVID-19. Kantar Media. https:/ / labur.eus/Huiv9

Kanungo, R.N. (1982). Measurement of job and work involvement. Journal of Applied Psychology, 67, 341-349.

Keating, G. (2012). Netflixed: The Epic Battle for America's Eyeballs. New York: The Penguin Group. 
Leon Frías, I. F. (2020). Netflix: El nuevo paradigma en la producción y distribución cinematográfica global y su incidencia en el cine latinoamericano: estudio del caso «Roma», de Alfonso Cuarón. Tesis de Maestría. Universidad San Martín de Porres, Perú.

Lotz, A. D. (2014). The television will be revolutionized. NYU Press.

Medina, M.; Herrero, M. \& Urgellés, A. (2017). Current and emerging issues in the audiovisual industry, vol. 1. John Wiley \& Sons.

Munita, R. G. H. (2016). La narrativa como método desencadenante y producción teórica en la investigación cualitativa. EMPIRIA. Revista de Metodología de las Ciencias Sociales, 34, 155-177.

Murolo, L. y Aon, L. (2018). Maratón en Netflix. House of cards entre la narrativa de la televisión y la web. Tram [p] as de la comunicación y la cultura, 82.

Neira, E. (2020). Lo que nos dejó2020 en la guerra del streaming (27 de diciembre de 2020). Business Insider. https:/ / www.businessinsider.es/nos-dejo-2020-guerra-streaming-779191

O'Malley, G. (2016). Netflix, YouTube Remain Leaders in Broadband Service MediaPost, June 22, 2016. https:/ / www.mediapost.com/publications/article/278806/netflix-youtuberemain-leaders-in-broadband-servi.html

Panda S. \& Pandey S. C. (2017). Binge watching and college students: Motivations and outcomes. Young Consumers, 18(4), 425-438.

Pozzi, S. (2016, 20 de enero). Netflix reduce el beneficio a la mitad por el coste de la expansión. El País. http://economia.elpais.com/economia/2016/01/19/ actualidad/1453237457 384873.html

PriceWaterHouseCoopers (2020). Perspectives from the Global Entertainment \& Media Outlook 2020-2024. perspectives.pdf

Rubenking, B.; Bracken, C. C.; Sandoval, J. \& Rister, A. (2018). Defining new viewing behaviours: What makes and motivates TV binge-watching? International Journal of Digital Television, 9(1), 69-85.

Small O. (2012). Reshaping the music distribution model: an iTunes opportunity. Journal of Media BusinessStudies, 9(4), 41-68.

Spangler, T. (16 de diciembre de 2019). Netflix breaks down results by region, showing strong Asia-Pacific, Europe Growth. Variety. https://variety.com/2019/digital/ news/netflix-breaks-down-results-by-region-showing-strong-asia-pacific-europegrowth-1203440307/

Soler, P. (1990). Investigación en marketing y publicidad. Bilbao: Deusto.

Statista (2020). Distribución porcentual de gasto en publicidad en México desde el 2018 hasta 2024, por medio de comunicación. https://es.statista.com/estadisticas/628296/porcentaje-degasto-en-publicidad-por-medio-mexico/

Stewart, D. W. \& Shamdasani, P. (2017). Online focus groups. Journal of Advertising, 46(1), 48-60.

Steyerl, H. (2009). In defense of the poor image. E-flux, n. 10, November. https:/ / www.e-flux. com/journal/10/61362/in-defense-of-the-poor-image/ 
Strangelove, M. (2015). Post-TV: Piracy, cord-cutting, and the future of television. University of Toronto Press.

Steiner, E. \& Xu, K. (2020). Binge-watching motivates change: Uses and gratifications of streaming video viewers challenge traditional TV research. Convergence, 26(1), 82-101.

Sweet, C. (2001). Designing and conducting virtual focus groups. Qualitative Market Research: An International Journal.

Sweney, M. (march 12, 2021). Netflix weighs up crackdown on password sharing. The guardian. https:// www.theguardian.com/media/2021/mar/12/netflix-weighs-up-crackdownon-password-sharing

Taylor, M. \& Kent, M. L. (2014). Dialogicengagement: Clarifying foundational concepts. Journal of Public Relations Research, 26(5), 384-398. https:/ / doi.org/10.1080/1062726X.2014.956106

Vonderau P. (2015). The politics of content aggregation. Television \& New Media, 16(8), 717-733.

Van Weezel, A. \& Benavides, C. (2010). Adoption of high definition television in Chile. Conference paper presented at 9th World Media Economic and Management Conference, Bogotá, Colombia. 